Recombinant vaccines now being evaluated in man follow one of three basic approaches: subunit vaccines, in which a cloned gene is used to produce large amounts of a specific antigen or to predict the amino acid sequence of a synthetic peptide; live, attenuated vaccines, in which recombinant techniques are employed to mutate crucial virulence or metabolic genes; and carrier vaccines, in which attenuated viral or bacterial strains serve as hosts for cloned genes expressing foreign antigens.

In this issue of Bio/Technology, Koji Nakayama and colleagues at Washington University (St. Louis, MO) describe a versatile and powerful bacterial carrier system that could be used in developing a variety of new vaccines.

Thus far, the vaccinia system has received the most attention as a carrier vaccine. Researchers have engineered the smallpox vaccine virus to express foreign antigens from a number of infectious agents, including those responsible for hepatitis, herpes, rabies, and malaria. As an alternate approach, scientists have turned to bacteria of the genus Salmonella. A serious problem with Salmonella as carriers of foreign antigens, however, has been the instability of recombinant plasmids in these strains. Nakayama and co-workers in the laboratory of Roy Curtiss III have solved this problem by constructing a balanced-lethal host-vector system in which survival of the bacterial host depends upon maintenance of the recombinant plasmid. The new approach does not use antibiotics or special growth media to retain the plasmid but instead exploits an essential bacterial metabolic pathway.

The researchers constructed Salmonella strains with a chromosomal deletion of the asd gene, which encodes aspartate $\beta$-semialdehyde dehydrogenase. This enzyme plays a role in the biosynthetic pathway for diaminopimelic acid (DAP), a key component of the peptidoglycan of the cell wall of Gram-negative bacteria. Salmonella with a deleted asd gene must maintain the proficient plasmidborne asd in order to avoid a "DAPless death." The vector now described by Nakayama utilizes this selective pressure to maintain foreign genes cloned under the control of a strong promoter.

Specifically, the scientists employ host S. typhimurium strains attenuated by deletion of genes encoding adenyl- ate cyclase and cyclic AMP receptor protein. Such strains, recently described by Curtiss and Sandra Kelly (Infection and Immunity 55:3042, 1987), exhibit a diminished ability to invade mesenteric lymph nodes and the spleen but are still capable of delivering antigens to the gut-associated lymphoid tissue. S. typhimurium attenuated in this manner are avirulent yet able to stimulate both humoral and cellular immune responses in animals. Similarly attenuated strains of the human pathogen, S. typhi, are under development for use in man. A variety of foreign antigens-including toxin subunits, fimbrial colonization factors, surface lipopolysaccharides, and Plasmodium circumsporozoite protein-have already been cloned and expressed in attenuated Salmonella strains.

Carrier vaccines, whether bacterial or viral, offer several advantages over subunit vaccines: Subunit products require adjuvants and multiple doses, and they are generally more expensive and less immunogenic than live vaccines.

-James B. Kaper

Kaper is chief of the Bacterial Genetics Section of the University of Maryland School of Medicine's Center for Vaccine Development.

\title{
DIRECT MONITORING OF FUNGAL OIL SYWTHESIS
}

\author{
IMAGE \\ UNAVAILABLE FOR \\ COPYRIGHT \\ REASONS
}

The use of microorganisms as an alternative to seeds for the production of fats and oils is becoming increasingly attractive, particularly in countries where conventional starting material is in short supply. As shown in these photographs, researchers under the direction of R. Sankaran at the Defence Food Research Laboratory (Mysore, India) have developed a rapid and simple staining technique to detect the onset (left), accumulation (center), and termination (right) of fat synthesis in the oil-producing fungus, Fusarium pallidoroseum. Using the lipid-soluble dye, Oil Red $O$ (which darkly stains hydrophobic lipids, and only weakly interacts with phospholipids) and Trypan Blue as a counterstain, the scientists were able to monitor oil synthesis in the fungal mycelium as accurately as by standard gravimetric techniques. In contrast to gravimetric determinations-which require harvesting, drying, and extracting the mycelium, and consume 12 or more hours-colorimetric estimates can be made in 30 minutes. 